\title{
Functions of limited accumulation
}

\author{
IMME VAN DEN BERG
}

\begin{abstract}
Measures on the real line may be decomposed into a regular, singular and atomic part. The objective of the present article is to provide analogous decompositions for a class of nonstandard, discrete functions defined on an infinitesimal discretization of the real line.
\end{abstract}

2010 Mathematics Subject Classification 03H05 (primary); 26A30, 46F30 (secondary)

Keywords: limited accumulation, atomic, singular and regular discrete functions, decomposition, nonstandard analysis, atomic

\section{Introduction}

Functions of limited accumulation are nonstandard, discrete functions defined on an infinitesimal discretization of the real line, such that their partial sums over discrete intervals of infinitesimal length are limited. In fact this local property of limited accumulation is semi-global, for it will be proved that also their partial sums over discrete intervals of limited length are limited. Functions of limited accumulation may be decomposed into a regular, singular and atomic part, imitating at in a discrete setting the well-known analogous decomposition of measures on the real line.

We consider five types of decompositions. Firstly, we identify atomic, singular and regular contributions to the discrete integral of a function of limited accumulation. Secondly, respectively thirdly, we identify internal and external subsets where the contributions are realized. The fourth decomposition concerns a decomposition of the function of limited accumulation itself, in an atomic, singular and regular function. The last decomposition links a given decomposition of the function of limited accumulation to a decomposition of its discrete primitive into a jump-function, a sort of discrete Cantor function - locally constant almost everywhere, but not globally constant - and a sort of discrete absolutely continuous function.

For classical results on the decomposition of measures we refer for example to Bartle [1]. Various nonstandard authors recognized the possibility to obtain decompositions of 
measures or alternatively distributions by means of internal functions, for instance Hurd and Loeb [11], Cartier and Perrin [7], Stroyan and Bayod [18], and Sousa Pinto [17]. Still a comprehensive treatment of the decomposition using uniquely discrete internal functions seems novel. Being devoted to discrete integration, in a sense the present article is a complement to the article on discrete differentiation (van den Berg [4]). Both articles attempt to share the spirit of simplicity due to the use of external concepts of the "radically elementary probability theory" of Nelson [16] and the "approximate analysis" of Callot [5].

Section 2 contains some preliminary remarks and notations. In Section 3 we define the functions of limited accumulation. We consider some examples, the most characteristic being a discrete Dirac function, a sort of discrete $\Delta$-function. We investigate some basic properties, like accumulation number, and gathering point and domain. Within the class of functions of limited accumulation we identify subclasses: atomic functions, functions of infinitesimal accumulation, singular and regular functions.

In Section 4 we investigate the behavior of the functions of limited accumulation under elementary operations. We prove that the discrete integral of a function of limited accumulation over the whole domain of definition is limited. We treat rather thoroughly the class of atomic functions, which have no counterpart in the classical theory of real functions.

The existence of the various types of decomposition of functions of limited accumulation is proved in Section 5 .

The setting of this article is the axiomatic form of nonstandard analysis IST of Nelson. Introductions and notations are contained in Diener and Diener [8], Robert [13], Diener and Reeb [9] and de Oliveira and van den Berg [10].

The article uses material from the masters thesis of Canelas [6] and a previous unpublished manuscript of the author; the notion of limited accumulation was also used in van den Berg [3]. I thank Jacques Bosgiraud (University Paris VIII) for careful reading of the manuscript and suggestions for improvement.

\section{Preliminaries}

In this article we consider functions defined on a discrete subset of $\mathbb{R}$, consisting of successive points at an infinitesimal distance. 
Definition 2.1 We let $\delta x$ always be a positive non-zero infinitesimal and $\mathbb{X}=\{k \delta x \mid k \in$ $\mathbb{Z}\}$. Let $a, b \in \mathbb{X}$ be limited with $a<b$. We define the near-interval $[a \ldots b]$ by

$$
[a \ldots b]=\{x \in \mathbb{X} \mid a \leq x \leq b\} .
$$

We define also

$$
[a \ldots b[=\{x \in \mathbb{X} \mid a \leq x<b\} .
$$

The set $\mathbb{X}$ is called an equidistant near-continuum in van den Berg [4]. We use here three dots to indicate near-intervals, instead of two. We will reserve the notation $[a . . b]$ for a near-interval with equally spaced points at distance $\sqrt{\delta x}$.

The choice to develop discrete integration on an equidistant near-continuum is by convenience. Mutatis mutandis a general near-continuum can be used; we recall here its definition. Let $\mathbb{Y} \subset \mathbb{R}$ be internal; it is called a near-continuum if it is the image of an equidistant near-continuum by a strictly monotone function $\varphi: \mathbb{X} \rightarrow \mathbb{R}$ of class $S^{0}$ (a definition of the property of being of class $S^{0}$ can also be found in [4]).

A simple measure on the internal subsets of a discrete interval is given by the counting measure, as follows.

Definition 2.2 Let $[a \ldots b]$ be a near-interval. Let $A \subseteq[a \ldots b]$ be internal. We define the measure $\lambda A$ of $A$ by

$$
\lambda A=\sharp A \cdot \delta x .
$$

Observe that $\lambda[a \ldots b[=b-a$, the length of the corresponding interval $[a, b[$ of $\mathbb{R}$.

We will use some basic properties of nonstandard analysis.

If $x$ is a limited real number, the shadow or standard part ${ }^{\circ} x$ is the unique standard real number such that ${ }^{\circ} x \simeq x$. If $X$ is an internal or external set, the standardization ${ }^{s t} X$ is the standard set having exactly the same standard elements as $X$. If $X$ is a standard set, we write ${ }^{\sigma} X$ the external set of all its standard elements.

The Extension Principle states that an external function with internal values defined only on the standard elements of a standard set has an extension which is an internal function defined on this set. The Cauchy Principle says that no external set is internal, it is often used to justify properties of overspill or permanence: an internal property valid on some external set is still valid somewhere outside this set. We recall that a galaxy $G$ is an external set of the form $G=\cup_{x \in{ }^{\sigma} X} A_{x}$, where $X$ is standard and $\left(A_{x}\right)_{x \in X}$ is an internal family of internal sets, and a halo $H$ is an external set of the form $H=\cap_{y \in{ }^{\sigma} Y} B_{y}$, where $Y$ is standard and $\left(B_{y}\right)_{y \in Y}$ is an internal family of internal sets; the latter notion 
generalizes the halo hal(x) of a real number $x$, which is the external set of all real numbers infinitely close to $x$. The Fehrele Principle says that no halo is a galaxy. It is also used to justify permanence of behavior, for example Robinson's Lemma [14] may be seen as a particular instance of this principle. The Lemma of Dominated Approximation (van den Berg [2]) is a consequence of Robinson's Lemma and says that if $f, g$ are Riemann-integrable real functions such that $\int_{a}^{b} f(x) d x \simeq \int_{a}^{b} g(x) d x$ for all limited $a, b \in \mathbb{R}$, and $|f|,|g|$ are bounded by a standard integrable function $h$, then $\int_{-\omega}^{\omega} f(x) d x \simeq \int_{-\omega}^{\omega} g(x) d x$ for all $\omega \simeq+\infty$. The lemma also holds for Riemann sums instead of integrals.

\section{Functions of limited accumulation: definitions and exam- ples}

We define the class of functions of limited accumulation and three definite subclasses: atomic functions, singular functions and regular functions. We introduce some related notions and present some examples.

Definition 3.1 Let $[a \ldots b]$ be a near interval. A function $\varphi:[a \ldots b] \rightarrow \mathbb{R}^{+}$is said to be of limited accumulation if $\sum_{y \leq x \leq z} \varphi(x) \delta x$ is limited for all $y, z \in[a \ldots b]$ with $y \simeq z, y \leq z$.

Clearly functions of class $S^{0}$ are of limited accumulation. We give here an example of a function of limited accumulation which takes unlimited values and is not $S$-continuous.

Example 3.2 Let $[a \ldots b]$ be a near interval and $c \in \mathbb{X}$ with $a<c<b$. Let $\Delta_{c}(x):[a \ldots b] \rightarrow \mathbb{R}$ be defined by

$$
\triangle_{c}(x)=\left\{\begin{array}{cl}
\frac{1}{\delta x} & x=c \\
0 & x \neq c .
\end{array}\right.
$$

Then $\Delta_{c}$ is a function of limited accumulation, since

$$
\sum_{a \leq x \leq b} \triangle_{c}(x)=\sum_{a \leq x<c} 0 \delta x+\frac{1}{\delta x} \delta x+\sum_{c<x \leq b} 0 \delta x=1 .
$$

We call $\triangle_{c}$ the discrete Dirac function associated to $c$.

Definition 3.3 Let $\varphi:[a \ldots b] \rightarrow \mathbb{R}^{+}$be a function of limited accumulation and $h \in\left[{ }^{\circ} a,{ }^{\circ} b\right]$ be standard. The accumulation number $\alpha_{h}$ of $\varphi$ at $h$ is defined by

$$
\alpha_{h}=\sup \left\{\left(\sum_{y \leq x \leq z} \varphi(x) \delta x\right) \mid y, z \simeq h\right\} .
$$


If $\alpha_{h}>0$, the point $h$ is called a gathering point of $\varphi$. The set $H={ }^{s t}\left\{h \mid \alpha_{h}>0\right\}$ is called the gathering domain of $\varphi$.

As for examples, let $[a \ldots b]$ be a near interval and $c \in \mathbb{X}$ with $a<c<b$. Then ${ }^{\circ} c$ is a gathering point of the discrete Dirac function $\triangle_{c}$, with accumulation number $\alpha^{\circ}{ }_{c}=1$. Now let $c_{1} \simeq c_{2} \in[a \ldots b], a<c_{1}<c_{2}<b$, and $f:[a \ldots b] \rightarrow \mathbb{R}^{+}$be defined by

$$
f(x)= \begin{cases}\frac{1}{2 \delta x} & x=c_{1} \text { or } x=c_{2} \\ 0 & x \neq c_{1}, c_{2} .\end{cases}
$$

Then $f$ is of limited accumulation, with gathering point ${ }^{\circ} c_{1}={ }^{\circ} c_{2}$ and accumulation number

$$
\alpha^{\circ} c_{c_{1}}=\sup \left\{{ }^{s t}\left(\sum_{a_{1} \leq x \leq b_{1}} h(x) \delta x\right) \mid a_{1} \simeq b_{1} \simeq c_{1}\right\}=\sup \left\{0, \frac{1}{2}, 1\right\}=1 .
$$

A third example is given by a very concentrated Gaussian function, as follows. Let $a \in \mathbb{X}^{+}$be appreciable and $g:[-a \ldots a] \rightarrow \mathbb{R}$ be defined by

$$
g(x)=\frac{e^{\frac{-x^{2}}{2 \sqrt{\delta x}}}}{\sqrt{2 \pi \sqrt{\delta x}}}
$$

To see that $g$ is of limited accumulation, put $\xi=x / \delta x^{1 / 4}$. Then $\delta x=\delta \xi \cdot \delta x^{1 / 4}$ and

$$
\sum_{-a \leq x \leq a} \frac{e^{\frac{-x^{2}}{2 \sqrt{\delta x}}}}{\sqrt{2 \pi \sqrt{\delta x}}} \delta x=\sum_{\frac{-a}{\delta x^{1 / 4}} \leq \xi \leq \frac{a}{\delta x^{1 / 4}}} \frac{e^{\frac{-\xi^{2}}{2}}}{\sqrt{2 \pi}} \delta \xi .
$$

It is easily seen by the Lemma of Dominated Approximation that

$$
\sum_{\frac{-a}{\delta x^{1 / 4} \leq \xi \leq \frac{a}{\delta x^{1 / 4}}}} \frac{e^{\frac{-\xi^{2}}{2}}}{\sqrt{2 \pi}} \delta \xi \simeq \int_{-\infty}^{+\infty} \frac{e^{\frac{-\eta^{2}}{2}}}{\sqrt{2 \pi}} d \eta=1
$$

This implies that $g$ is of limited accumulation. Further, let $\omega$ be unlimited such that $\omega \delta x^{1 / 4} \simeq 0$. Then

$$
\sum_{-\omega \delta x^{1 / 4} \leq x \leq \omega \delta x^{1 / 4}} \frac{e^{\frac{-x^{2}}{2 \sqrt{\delta x}}}}{\sqrt{2 \pi \sqrt{\delta x}}} \delta x=\sum_{-\omega \leq \xi \leq \omega} \frac{e^{\frac{-\xi^{2}}{2}}}{\sqrt{2 \pi}} \delta \xi \simeq \int_{-\omega}^{\omega} \frac{e^{\frac{-\eta^{2}}{2}}}{\sqrt{2 \pi}} d \eta \simeq 1,
$$


so 0 is a gathering point of $g$. Its accumulation number is given by

$$
\begin{aligned}
\alpha_{0} & =\sup \left\{\left(\sum_{y \leq x \leq z} \frac{e^{\frac{-x^{2}}{2 \sqrt{\delta x}}}}{\sqrt{2 \pi \sqrt{\delta x}}} \delta x\right) \mid y, z \simeq 0\right\} \\
& =\sup \left\{\left(\sum_{\frac{y}{\delta x^{1 / 4} \leq \xi \leq \frac{z}{\delta x^{1 / 4}}}} \frac{e^{\frac{-\xi^{2}}{2}}}{\sqrt{2 \pi}} \delta \xi\right) \mid y, z \simeq 0\right\} \\
& =\sup \left\{\left(\int_{y / \delta x^{1 / 4}}^{\int^{s t}} \frac{e^{\frac{-\eta^{2}}{2}}}{\sqrt{2 \pi}} d \eta\right) \mid y, z \simeq 0\right\} \\
& =\sup { }^{s t}\left\{{ }^{\circ} w \mid w \in[0,1]\right\}=\sup [0,1]=1 .
\end{aligned}
$$

We define now two stronger notions. The second is rather common within nonstandard analysis.

Definition 3.4 Let $[a \ldots b]$ be a near interval. A function $\varphi:[a \ldots b] \rightarrow \mathbb{R}^{+}$is said to be of infinitesimal accumulation if $\sum_{y \leq x \leq z} \varphi(x) \delta x \simeq 0$ for all $y, z \in[a \ldots b]$ with $y \simeq z$, $y \leq z$

Definition 3.5 (See also Hurd and Loeb [11], Cartier and Perrin [7].) Let [a..b] be a near interval. Let $D \subseteq[a \ldots b]$. A function $\varphi:[a \ldots b] \rightarrow \mathbb{R}^{+}$is said to be $S$-integrable or regular on $D$ if for all internal subsets $N \subseteq D$,

$$
\lambda N \simeq 0 \Rightarrow \sum_{x \in N} \varphi(x) \delta x \simeq 0 .
$$

Clearly an $S$-integrable function is of infinitesimal accumulation. Obvious examples of $S$-integrable functions are limited functions and in particular functions of class $S^{0}$. Still, an $S$-integrable function may take unlimited values, as is shown by the function $\varphi(x):[-1 \ldots 1] \rightarrow \mathbb{R}^{+}$defined by

$$
\varphi(x)=\left\{\begin{array}{cc}
\sqrt{\frac{1}{\delta x}} & x=0 \\
0 & x \neq 0 .
\end{array}\right.
$$

The next example exhibits a function of infinitesimal accumulation which is not $S$-integrable. 
Example 3.6 Assume for convenience that $\frac{1}{\sqrt{\delta x}} \in \mathbb{N}$. Define $f:\left[0 \ldots 1\left[\rightarrow \mathbb{R}^{+}\right.\right.$by

$$
\begin{gathered}
f(x)=\left\{\begin{array}{cl}
\frac{1}{\sqrt{\delta x}} & \frac{x}{\sqrt{\delta x}} \in \mathbb{N} \\
0 & \text { else. }
\end{array}\right. \\
\text { Let } \eta=\left\{0, \sqrt{\delta x}, 2 \sqrt{\delta x}, 3 \sqrt{\delta x}, \ldots,\left(\frac{1}{\sqrt{\delta x}}-1\right) \sqrt{\delta x}\right\} . \text { Then } \\
\lambda \eta=\frac{1}{\sqrt{\delta x}} \delta x=\sqrt{\delta x} \simeq 0
\end{gathered}
$$

and

$$
\sum_{x \in \eta} f(x) \delta x=\sum_{x \in \eta} \sqrt{\delta x}=\frac{1}{\sqrt{\delta x}} \sqrt{\delta x}=1
$$

A discrete Dirac function is an example of a function of limited accumulation which is not of infinitesimal accumulation.

The notion of function of limited accumulation has been defined for positive functions. The notion may not be extended as such to alternate functions without undesirable consequences. This is illustrated by the next example.

Let $f:[0 \ldots 1] \rightarrow \mathbb{R}$ be defined by

$$
f(x)=\left\{\begin{array}{cc}
\frac{1}{\delta x} & \frac{x}{\delta x} \text { even } \\
-\frac{1}{\delta x} & \frac{x}{\delta x} \text { odd }
\end{array}\right.
$$

Let $y, z$ be such that $y<z, y \simeq z$. Then $-1 \leq \sum_{y \leq x \leq z} f(x) \delta x \leq 1$, so $f$ is of limited accumulation. But $f{ }^{+}$and $f-$ are not of limited accumulation. Indeed, if $z-y=k \delta x$ with $k \simeq+\infty$ even, one has

$$
\sum_{y \leq x \leq z} f^{+}(x) \delta x=\left[\frac{1}{\delta x}\left(\frac{z-y}{2 \delta x}\right) \delta x\right]=\frac{k}{2},
$$

which is unlimited. In the same way we find $\sum_{y \leq x \leq z} f-(x) \delta x=-\frac{k}{2} \simeq-\infty$.

We might consider adaptations of the notion of $\varphi$ being of limited accumulation, by applying it to $|\varphi|$, or by asking that $\sum_{x \in \eta} \varphi(x) \delta x$ is limited for any set $\eta \subset \mathbb{X}$ of infinitesimal measure, but we do not pursue this here.

Next to regular functions we distinguish two more types of functions of limited accumulation. Our goal will be to show that every function of limited accumulation can be decomposed into functions of the types in question. 
Definition 3.7 Let $\varphi:[a \ldots b] \rightarrow \mathbb{R}^{+}$be a function of limited accumulation. The function $\varphi$ will be called atomic if there exists an internal set $C \subseteq[a \ldots b]$ of infinitesimal measure such that $\varphi(x)=0$ for $x \in[a \ldots b] \backslash C$ and such that $\sum_{x \in D} \varphi(x) \delta x \simeq 0$ for every internal set $D \subseteq C$ such that $D \cap$ hal(h) $=\emptyset$ for every gathering point $h$ of $\varphi$.

Examples of atomic functions are the discrete Dirac-functions, standard finite sums of them, and the function $g$ defined in (1), when restricted to an interval of infinitesimal length. Observe that a function of limited accumulation without gathering points is necessarily of infinitesimal accumulation.

Definition 3.8 Let $\varphi:[a \ldots b] \rightarrow \mathbb{R}^{+}$be a function of infinitesimal accumulation. The function $\varphi$ will be called singular if there exists a set $\eta$ of infinitesimal measure such that $\varphi(x)=0$ for $x \in[a \ldots b] \backslash \eta$.

The function $\varphi$ defined in Example 3.6 is an example of a singular function.

\section{Properties of functions of limited accumulation}

We will always consider non-negative functions defined on a given near-interval $[a \ldots b]$. We show that functions of limited accumulation have a particular bound. As a consequence the class of functions of limited accumulation is closed under addition, but not under multiplication and discrete differentiation. Then we turn to discrete integrals of functions of limited accumulation. We prove a fundamental property of functions of limited accumulation: the total sum over the near-interval $[a \ldots b]$ is limited. This implies that the discrete primitive of a function of limited accumulation is of limited accumulation. We investigate in particular the properties of the gathering points with respect to the discrete integral.

Proposition 4.1 If $f$ is a function of limited accumulation, it is bounded by $\frac{c}{\delta x}$ for some limited $c$.

Proof Suppose not. Then by the Cauchy Principle there exists $x_{0} \in[a \ldots b]$ such that $f\left(x_{0}\right) \delta x \simeq \infty$. Then $\sum_{x_{0} \leq x<x_{0}+\delta x} f(x) \delta x=f\left(x_{0}\right) \delta x$ is unlimited. So we have a contradiction.

Proposition 4.2 The sum of two functions of limited accumulation is a function of limited accumulation. 
Proof Let $f$ and $g$ be two functions of limited accumulation and $y, z \in[a \ldots b]$ be such that $y \leq z, y \simeq z$. Then

$$
\sum_{y \leq x \leq z}(f(x)+g(x)) \delta x=\sum_{y \leq x \leq z} f(x) \delta x+\sum_{y \leq x \leq z} g(x) \delta x,
$$

which is limited, being the sum of two limited numbers.

Proposition 4.3 The product of two functions of limited accumulation is not necessarily a function of limited accumulation.

Proof A counterexample is given by the square of the discrete Dirac function $\Delta_{0}$. Indeed, let $y, z \in \mathbb{X}$ be such that $y<0, z>0$ and $y \simeq z$. Then

$$
\sum_{y \leq x \leq z} \Delta_{0}^{2}(x) \delta x=\Delta_{0}^{2}(0) \delta x=\frac{1}{\delta x^{2}} \delta x=\frac{1}{\delta x},
$$

which is unlimited.

Proposition 4.4 The discrete derivative of a discrete Dirac function is not a function of limited accumulation.

Proof Let $y \in[a \ldots b[$. Then

$$
\frac{\delta \Delta_{y}(y-\delta x)}{\delta x}=\frac{\Delta_{y}(y)-\Delta_{y}(y-\delta x)}{\delta x}=\frac{\frac{1}{\delta x}-0}{\delta x}=\frac{1}{\delta x^{2}},
$$

which is not of the form $c / \delta x$ with limited $c$.

We will now study discrete primitives and integrals of functions of limited accumulation.

Theorem 4.5 Let $\varphi$ be a function of limited accumulation. Let $I=\sum_{a \leq x<b} \varphi(x) d x$. Then $I$ is limited.

Proof Let $G$ be the external set of all $\epsilon \geq 0$ such that $\epsilon$ is the length of a discrete subinterval $\eta$ of $\left[a \ldots b\right.$ [ and $\sum_{x \in \eta} \varphi(x) \delta x$ is limited. Then $G$ is a galaxy or is internal. Clearly $G$ contains the halo, say $H$, formed by all multiples of $\delta x$ which are infinitesimal. One has $H \subseteq G$ by Definition 3.1, hence $H \varsubsetneqq G$ by the Fehrele Principle or the Cauchy Principle. Let $\epsilon \in G \backslash H$, then $\epsilon$ is appreciable. Hence there exists a standard $n \in \mathbb{N}$ and $x_{0}, x_{1}, \ldots, x_{n} \in[a \ldots b]$ such that $x_{0}=a, x_{0}<x_{1}<\ldots<x_{n}, x_{n}=b$ and $x_{i+1}-x_{i} \leq \epsilon$, for all $i$ with $0 \leq i \leq n-1$. Let

$$
M=\max \left\{\sum_{y \leq x \leq y+\epsilon} \varphi(x) \delta x \mid a \leq y, y+\epsilon \leq b\right\} .
$$


Then $M$ is limited. Hence

$$
I=\sum_{a \leq x<b} \varphi(x) \delta x=\sum_{i=0}^{n-1}\left(\sum_{x_{i} \leq x<x_{i+1}} \varphi(x) \delta x\right) \leq \sum_{i=0}^{n-1} M=n M
$$

is limited.

Definition 4.6 Let $\varphi:[a \ldots b] \rightarrow \mathbb{R}^{+}$be a function. The function $\Phi:\left[a \ldots b\left[\rightarrow \mathbb{R}^{+}\right.\right.$ given by $\Phi(x)=\sum_{a \leq y<x} \varphi(y) \delta x$ will be called its discrete primitive.

We derive the following consequence of Theorem 4.5.

Proposition 4.7 The discrete primitive of a function of limited accumulation is a limited non-decreasing function.

Proof Let $\varphi:[a \ldots b] \longrightarrow \mathbb{R}^{+}$be of limited accumulation and $\Phi$ its discrete primitive. Then $\Phi$ is non-decreasing. Moreover $\Phi(a)=0$, and $\Phi(b)$ is limited by Theorem 4.5. Hence $\Phi$ is limited.

Discrete primitives of functions of limited accumulation are functions of limited steps in the sense of Diener and Reeb [9]: these are discrete functions such that the difference of two successive values is at most limited. The steps of the discrete primitives which are truly limited and not infinitesimal correspond to the gathering points of the functions of limited accumulation.

We study now the behavior of a function of limited accumulation with respect to its gathering points.

Proposition 4.8 Let $\varphi$ be a function of limited accumulation. Let $h$ be a gathering point of $\varphi$ and $\alpha_{h}$ be the accumulation number of $\varphi$ at $h$. Then there exist $y, z \simeq h$ with $y \leq z$ such that for all $\eta, \zeta \simeq h$ with $\eta \leq y$ and $\zeta \geq z$

$$
\sum_{\eta \leq x \leq \zeta} \varphi(x) \delta x \simeq \alpha_{h}
$$

Proof Let $\eta, \zeta \simeq h$ be such that $\eta \leq \zeta$. We show firstly that

$$
\sum_{\eta \leq x \leq \zeta} \varphi(x) \delta x \lesssim \alpha_{h}
$$


We have

$$
\begin{aligned}
\sum_{\eta \leq x \leq \zeta} \varphi(x) \delta x & \simeq{ }^{\circ}\left(\sum_{\eta \leq x \leq \zeta} \varphi(x) \delta x\right) \\
& \leq \sup ^{s t}\left\{\left(\sum_{\beta \leq x \leq \gamma} \varphi(x) \delta x\right) \mid \beta, \gamma \simeq h, \beta \leq \gamma\right\} \\
& =\alpha_{h} .
\end{aligned}
$$

This implies (4).

Secondly we show that there exist $y, z \simeq h, y \leq z$ such that

$$
\sum_{y \leq x \leq z} \varphi(x) \delta x \gtrsim \alpha_{h}
$$

Let $n \in \mathbb{N}$ be standard. There exist $\beta, \gamma \in[a \ldots b$ [ such that $\beta<\gamma, \beta \simeq h \simeq \gamma$ and

$$
\sum_{\beta \leq x \leq \gamma} \varphi(x) \delta x>\alpha_{h}-\frac{1}{n} .
$$

By the Extension Principle, there exist internal sequences $\left(\beta_{n}\right)_{n \in \mathbb{N}}$ and $\left(\gamma_{n}\right)_{n \in \mathbb{N}}$ such that

$$
\sum_{\beta_{n} \leq x \leq \gamma_{n}} \varphi(x) \delta x>\alpha_{h}-\frac{1}{n}
$$

for all standard $n \in \mathbb{N}$. By Robinson's Lemma and the Cauchy Principle there exists unlimited $\nu \in \mathbb{N}$ such that still $\beta_{\nu} \simeq h \simeq \gamma_{\nu}$, and (6) holds for $n=\nu$. Then

$$
\sum_{\beta_{\nu} \leq x \leq \gamma_{\nu}} \varphi(x) \delta x \gtrsim \alpha_{h}
$$

Put $y=\beta_{\nu}$ and $z=\gamma_{\nu}$. Then (3) follows from (7) and (4).

Example 4.9 Let $\varphi:[a \ldots b] \rightarrow \mathbb{R}^{+}$be defined by

$$
\left\{\begin{array}{l}
\varphi(0)=\frac{1}{\delta x} \\
\varphi(\delta x)=\delta x \\
\varphi(-\delta x)=\delta x \\
\varphi(x)=0 \quad \text { if } x \notin\{-\delta x, 0, \delta x\} .
\end{array}\right.
$$

Then $\varphi$ is atomic, with accumulation number $\alpha_{0}=1$. Putting $y=-\delta x, z=\delta x$ we have for all $\eta, \zeta \simeq 0$ with $\eta \leq y$ and $\zeta \geq z$

$$
\sum_{\eta \leq x \leq \zeta} \varphi(x) \delta x=\delta x \cdot \delta x+\frac{1}{\delta x} \cdot \delta x+\delta x \cdot \delta x=1+2 \delta x^{2} \simeq 1 .
$$


Example 4.10 Consider the function $g$ defined in (1). Applying the substitution $x=y \cdot(\delta x)^{1 / 4}$ we derive from (2) with $\omega=\delta x^{-1 / 8}$ that

$$
\sum_{-\delta x^{1 / 8} \leq x \leq \delta x^{1 / 8}} \frac{e^{\frac{-x^{2}}{2 \sqrt{\delta x}}}}{\sqrt{2 \pi \sqrt{\delta x}}} \delta x \simeq 1 .
$$

Hence with $\eta, \zeta \simeq 0, \eta \leq-\delta x^{1 / 8}$ and $\zeta \geq \delta x^{1 / 8}$, also

$$
\sum_{\eta \leq x \leq \zeta} \frac{e^{\frac{-x^{2}}{2 \sqrt{\delta x}}}}{\sqrt{2 \pi \sqrt{\delta x}}} \delta x \simeq 1 .
$$

As a consequence of Theorem 4.5 and Proposition 4.8 we obtain that the external set of gathering points of a function of limited accumulation is externally countable, ie in one-to-one correspondence with (a subset of) the external set of standard elements of $\mathbb{N}$.

Theorem 4.11 The external set of gathering points of a function of limited accumulation is at most externally countable.

Proof Let $\varphi:[a \ldots b] \longrightarrow \mathbb{R}^{+}$be a function of limited accumulation and $\Phi$ its discrete primitive. Let $h$ be a gathering point of $\varphi$. By Proposition 4.8 there exist $y, z \in[a \ldots b$ [ with $y, z \simeq h$ and $y<z$ such that $\Phi(y) \lesssim \Phi(z)$. Now $\Phi(y)$ and $\Phi(z)$ are limited by Proposition 4.7. Then there exists a standard rational number $q$ such that ${ }^{\circ} \Phi(y)<q<{ }^{\circ} \Phi(z)$. Applying the Standardization Principle we may associate a standard rational number $q_{h}$ to every gathering point $h$ of $\varphi$; because $\Phi$ is non-decreasing, different gathering points correspond to different standard rational numbers. Because the external set of standard rational numbers is externally countable, the external set of gathering points is at most externally countable.

\section{Decompositions}

We consider five types of decompositions. In Subsection 5.1 we distinguish atomic, singular and regular contributions to the discrete integral of a function of limited accumulation. In Subsection 5.2 the domain is decomposed into internal, respectively external, subdomains where these contributions are realized. In Subsection 5.3 we decompose the function of limited accumulation itself, into an atomic, singular and regular function. In the final Subsection 5.4 we show that this decomposition of a function of limited accumulation corresponds to a decomposition of its discrete primitive into a kind of jump-function, a sort of discrete Cantor-function and a sort of discrete absolutely continuous function. 


\subsection{Contributions to the discrete integral}

Again we consider functions $\varphi$ of limited accumulation defined on a near-interval $[a \ldots b]$. By Theorem 4.11 the gathering domain $H$ of $\varphi$ may be arranged into a sequence. With the help of $H$ we identify three definite types of contribution to the value of the discrete integral $I=\sum_{a \leq x<b} \varphi(x) \delta x$ of $\varphi$.

Notation 5.1 Let $\varphi$ be a function of limited accumulation. We will write the gathering domain of $\varphi$ in the form $H=\left\{h_{n} \mid n \leq m\right\}$ in case $H$ has $m$ elements, for some standard $m \in \mathbb{N}$, and in case $H$ is infinite we write $H=\left\{h_{n} \mid n \in \mathbb{N}\right\}$, where $h_{n}$ is a gathering point of $\varphi$ for every standard $n \in \mathbb{N}$. Whenever $h_{n}$ is an gathering point of $\varphi$, we let $\alpha_{h_{n}}$ be the accumulation number associated to $h_{n}$. If $H$ has $m$ elements for some standard $m \in \mathbb{N}$, we put $\alpha_{h_{n}}=0$ for $n>m$.

Definition 5.2 Let $\varphi$ be a function of limited accumulation. Let $\left(\alpha_{h_{n}}\right)_{n \in \mathbb{N}}$ be the standardized of the (possibly) external sequence $\left(\alpha_{h_{n}}\right)_{\text {st } n \in \mathbb{N}}$ of its accumulation numbers. Then the series A defined by

$$
A=\sum_{n=0}^{\infty} \alpha_{h_{n}}
$$

is called the atomic contribution of $\varphi$ to $I$.

Definition 5.3 Let $\varphi$ be a function of limited accumulation. We define the singular contribution $S$ of $\varphi$ to $I$ by

$$
S=\sup \left\{\left(\sum_{x \in N} \varphi(x) \delta x\right) \mid N \subset[a \ldots b] \backslash \bigcup_{h \in{ }^{\sigma} H} \operatorname{hal}(\mathrm{h}), \mathrm{N} \text { internal, } \lambda \mathrm{N} \simeq 0\right\} .
$$

Definition 5.4 Let $\varphi$ be a function of limited accumulation. We define the regular contribution $R$ of $\varphi$ to $I$ by

$$
R=\sup \left\{{ }^{s t}\left(\sum_{x \in D} \varphi(x) \delta x\right) \mid D \subset[a \ldots b] \text { internal, } \varphi S \text {-integrable on } D\right\} .
$$

In order to justify the definitions we start by showing that the series $\sum_{n=0}^{\infty} \alpha_{h_{n}}$ converges indeed.

Lemma 5.5 Let $\varphi$ be a function of limited accumulation. Let $m \in \mathbb{N}$ be standard and $h_{0}, \ldots, h_{m}$ be accumulation points of $\varphi$. Then there exist disjoint internal intervals $J_{0}, \ldots, J_{m} \subseteq[a \ldots b]$ such that $J_{0} \subset \operatorname{hal}\left(\mathrm{h}_{0}\right), \ldots, J_{m} \subset \operatorname{hal}\left(\mathrm{h}_{\mathrm{m}}\right)$ and $\sum_{n=0}^{m} \alpha_{h_{n}} \simeq$ $\sum_{n=0}^{m} \sum_{x \in J_{n}} \varphi(x) \delta x$. 
The lemma is an immediate consequence of Proposition 4.8.

Theorem 5.6 Let $\varphi$ be a function of limited accumulation. Then its atomic contribution $\sum_{n=0}^{\infty} \alpha_{h_{n}}$ converges.

Proof It is only needed to consider the case where $H$ is infinite. Let $m \in \mathbb{N}$ be standard. By Lemma 5.5 there exist internal intervals $J_{0}, \ldots, J_{m} \subseteq[a \ldots b]$ such that $J_{0} \subset$ hal $\left(\mathrm{h}_{0}\right)$, $\ldots, J_{m} \subset \operatorname{hal}\left(\mathrm{h}_{\mathrm{m}}\right)$ and $\sum_{n=0}^{m} \alpha_{h_{n}} \simeq \sum_{n=0}^{m} \sum_{x \in J_{n}} \varphi(x) \delta x \leq I$. Then $\sum_{n=0}^{m} \alpha_{h_{n}} \leq{ }^{\circ} I$ for all standard $m \in \mathbb{N}$. By Transfer $\sum_{n=0}^{m} \alpha_{h_{n}} \leq{ }^{\circ} I$ for all $m \in \mathbb{N}$. Hence $\sum_{n=0}^{\infty} \alpha_{h_{n}}$ converges, the sequence of its partial sums being non-decreasing.

Theorem 5.7 Let $\varphi$ be a function of limited accumulation.

(1) There exist $\nu \in \mathbb{N}$ and an internal sequence $\left(J_{n}\right)_{n \leq \nu}$ of disjoint intervals such that, with $C=\bigcup_{n \leq \nu} J_{n}$,

(a) For every standard $n \in \mathbb{N}$ such that $\alpha_{h_{n}} \neq 0$ it holds that $J_{n} \subset$ hal $\left(\mathrm{h}_{\mathrm{n}}\right)$ and $\sum_{x \in J_{n}} \varphi(x) \delta x \simeq \alpha_{h_{n}}$.

(b) $\lambda C \simeq 0$.

(c) $\sum_{x \in C} \varphi(x) \delta x \simeq A$.

(2) If $\nu^{\prime} \in \mathbb{N}$ and $\left(J_{n}^{\prime}\right)_{n \leq \nu^{\prime}}$ is an internal sequence with union $C^{\prime}=\bigcup_{n \leq \nu^{\prime}} J_{n}^{\prime}$, having the same properties, one has $\sum_{x \in C \Delta C^{\prime}} \varphi(x) \delta x \simeq 0$.

(3) If $I \subseteq C$ is internal and $I \cap \cup\left\{\operatorname{hal}\left(\mathrm{h}_{\mathrm{n}}\right) \mid \operatorname{stn} \in \mathbb{N}, \alpha_{\mathrm{h}_{\mathrm{n}}} \neq 0\right\}=\emptyset$, one has $\sum_{x \in I} \varphi(x) \delta x \simeq 0$.

Proof (1) If $H$ is standard finite, say of the form $\left\{h_{0}, \ldots, h_{m}\right\}$ with standard $m$, by Proposition 4.8 and Lemma 5.5 there exist disjoint internal intervals $J_{0}, \ldots, J_{m} \subseteq$ $[a \ldots b]$ such that $J_{0} \subset \operatorname{hal}\left(\mathrm{h}_{0}\right), \ldots, J_{m} \subset \operatorname{hal}\left(\mathrm{h}_{\mathrm{m}}\right)$ and $\sum_{x \in J_{n}} \varphi(x) \delta x \simeq \alpha_{h_{n}}$ for all $n$ with $1 \leq n \leq m$. Let $C=\bigcup_{1 \leq n \leq m} J_{n}$. Then $\lambda C=\lambda\left(\bigcup_{n \leq m} J_{n}\right)=\sum_{n=0}^{m} \lambda J_{n} \simeq$ 0 and $\sum_{x \in C} \varphi(x) \delta x=\sum_{n=0}^{m} \sum_{x \in J_{n}} \varphi(x) \delta x \simeq \sum_{n=0}^{m} \alpha_{h_{n}}=A$.

Now suppose that $H=\left\{h_{n} \mid n \in \mathbb{N}\right\}$ is infinite. By the above method we obtain an external sequence $\left(J_{n}\right)_{s t n \in \mathbb{N}}$ of internal disjoint intervals of infinitesimal length such that $J_{n} \subset \operatorname{hal}\left(\mathrm{h}_{\mathrm{n}}\right), \sum_{x \in J_{n}} \varphi(x) \delta x \simeq \alpha_{h_{n}}$ for all standard $n$, 
$\sum_{n=0}^{m} \sum_{x \in J_{n}} \varphi(x) \delta x \simeq \sum_{n=0}^{m} \alpha_{h_{n}}$ for all standard $m$, and $\lambda\left(\bigcup_{1 \leq n \leq m} J_{n}\right) \simeq 0$. By the Extension Principle there exists an internal sequence $\left(J_{n}\right)_{n \in \mathbb{N}}$ extending the external sequence $\left(J_{n}\right)_{s t n \in \mathbb{N}}$. By the Cauchy Principle there exists $\nu \in \mathbb{N}$ such that all intervals $J_{n}, n \leq \nu$, are disjoint. Also, applying Robinson's Lemma we may assume, with $C=\bigcup_{n \leq \nu} J_{n}$, that still $\lambda C \simeq 0$ and $\sum_{x \in C} \varphi(x) \delta x=\sum_{n=0}^{\nu} \sum_{x \in J_{n}} \varphi(x) \delta x \simeq \sum_{n=0}^{\nu} \alpha_{h_{n}}$. By Theorem 5.6 the series $\sum_{n=0}^{\infty} \alpha_{h_{n}}$ converges to $A$, and because it is standard $\sum_{n=0}^{\nu} \alpha_{h_{n}} \simeq A$. Hence $\sum_{x \in C} \varphi(x) \delta x \simeq A$.

(2) Assume $C^{\prime}=\bigcup_{n \leq \nu^{\prime}} J_{n}^{\prime}$ has the same properties. It follows from Proposition 4.8 that $\sum_{x \in J_{n} \Delta J_{n}^{\prime}} \varphi(x) \delta x \simeq 0$ for all standard $n$. It follows from the Cauchy Principle and Robinson's Lemma that there exists $\mu \simeq+\infty$ with $\mu \leq \min \left(\nu, \nu^{\prime}\right)$ such that $J_{m} \bigcap J_{n}^{\prime}=\emptyset$, once $m \neq n, m, n \leq \mu$, and

$$
\sum_{n \leq \mu}\left(\sum_{x \in J_{n} \Delta J_{n}^{\prime}} \varphi(x) \delta x\right) \simeq 0
$$

Now

$$
C \Delta C^{\prime} \subseteq\left(\bigcup_{n \leq \mu} J_{n} \Delta J_{n}^{\prime}\right) \cup\left(\bigcup_{\mu \leq n \leq \nu} J_{n}\right) \cup\left(\bigcup_{\mu \leq n \leq \nu^{\prime}} J_{n}^{\prime}\right)
$$

Because

$$
\sum_{0 \leq n \leq \mu}\left(\sum_{x \in J_{n}} \varphi(x) \delta x\right) \simeq \sum_{0 \leq n \leq \mu}\left(\sum_{x \in J_{n}^{\prime}} \varphi(x) \delta x\right) \simeq A
$$

one has

$$
\sum_{\mu \leq n \leq \nu}\left(\sum_{x \in J_{n}} \varphi(x) \delta x\right) \simeq 0
$$

and

$$
\sum_{\mu \leq n \leq \nu^{\prime}}\left(\sum_{x \in J_{n}^{\prime}} \varphi(x) \delta x\right) \simeq 0 .
$$

Combining (9), (8), (10) and (11), we prove that $\sum_{x \in C \Delta C^{\prime}} \varphi(x) \delta x \simeq 0$. 
(3) Let $I \subseteq C$ be internal and $I \cap \cup\left\{\operatorname{hal}\left(\mathrm{h}_{\mathrm{n}}\right) \mid \operatorname{stn} \in \mathbb{N}, \alpha_{\mathrm{h}_{\mathrm{n}}} \neq 0\right\}=\emptyset$. Put $C^{\prime}=C \backslash I$ and $J_{n}^{\prime}=J_{n} \backslash I$ for all $n \leq \nu$. Then $J_{n}^{\prime}=J_{n}$ for all standard $n \in \mathbb{N}$. Then Part 2 implies that $\sum_{x \in I} \varphi(x) \delta x \simeq 0$.

Observe that the restriction of $\varphi$ to the set $C$ of the above theorem is an atomic function.

Next theorem relates the singular contribution and the regular contribution to the accumulated contribution to the value of the discrete integral.

Theorem 5.8 Let $\varphi$ be a function of limited accumulation. Let $C$ as been given by Theorem 5.7.

(1) There exists an internal set $M \subseteq[a \ldots b] \backslash C$, with $\lambda M \simeq 0$, such that $\varphi$ is a function of infinitesimal accumulation on $M$ and $\sum_{x \in M} \varphi(x) \delta x \simeq S$. If $M^{\prime}$ has the same properties, with respect to a set $C^{\prime}$ given by Theorem 5.7, one has $\sum_{x \in M \triangle M^{\prime}} \varphi(x) \delta x \simeq 0$.

(2) There exists an internal set $Q \subseteq[a \ldots b] \backslash(C \cup M)$, with $\lambda Q \simeq b-a$, such that $\varphi_{\mid Q}$ is $S$-integrable and $\sum_{x \in Q} \varphi(x) \delta x \simeq R$. If $Q^{\prime}$ has the same properties with respect to a set $C^{\prime}$ given by Theorem 5.7 and a set $M^{\prime}$ given by Part 1, one has $\sum_{x \in Q \triangle Q^{\prime}} \varphi(x) \delta x \simeq 0$.

Proof Let $C \subseteq[a \ldots b]$ be an internal set such that $\lambda C \simeq 0$ and $\sum_{x \in C} \varphi(x) \delta x \simeq A$, such as given by Theorem 5.7.

(1) For every standard $n \in \mathbb{N}$ there exists an internal set $N_{n} \subseteq[a \ldots b] \backslash \bigcup_{h \in{ }^{\sigma} H}$ hal(h) such that $\lambda N_{n} \simeq 0$ and $S \geq \sum_{x \in N_{n}} \varphi(x) \delta x \geq S-\frac{2}{n}$. For such $n$, put $M_{n}=N_{n} \backslash C$. Because $\left(N_{n} \cap C\right) \cap \bigcup_{h \in{ }^{\sigma} H} \operatorname{hal}(\mathrm{h})=\emptyset$, Theorem 5.7 .3 implies that $\sum_{x \in N_{n} \cap C} \varphi(x) \delta x \simeq 0$. Hence $S \geq \sum_{x \in M_{n}} \varphi(x) \delta x=\sum_{x \in N_{n}} \varphi(x) \delta x-$ $\sum_{x \in N_{n} \cap C} \varphi(x) \delta x \geq S-\frac{1}{n}$. By the Extension Principle there exists an internal sequence $\left(M_{n}\right)_{n \in \mathbb{N}}$ extending the external sequence $\left(M_{n}\right)_{n \in \sigma} \mathbb{N}$. Applying Robinson's Lemma and the Cauchy Principle we see that there exists $\nu \simeq \infty$ such that still $\lambda M_{\nu} \simeq 0, M_{\nu} \subseteq[a \ldots b] \backslash C$ and $S \geq \sum_{x \in M_{\nu}} \varphi(x) \delta x \geq S-\frac{1}{\nu}$. Put $M=M_{\nu}$. Because $M \subseteq[a \ldots b] \backslash C$, the function $\varphi$ is of infinitesimal accumulation on $M$. Moreover, $\lambda M \simeq 0$ and $\sum_{x \in M} \varphi(x) \delta x \simeq S$.

Assume that $M^{\prime}$ has the same properties, as prescribed. Because $\lambda(M \cup$ 
$\left.M^{\prime}\right) \simeq 0$, the definition of $S$ implies that $\sum_{x \in M \cup M^{\prime}} \varphi(x) \delta x \lesssim S$, hence $\sum_{x \in M \cup M^{\prime}} \varphi(x) \delta x \simeq S$. So

$$
S \lesssim \sum_{x \in M} \varphi(x) \delta x+\sum_{x \in M^{\prime} \backslash M} \varphi(x) \delta x \simeq \sum_{x \in M \cup M^{\prime}} \varphi(x) \delta x \simeq S,
$$

which implies that $\sum_{x \in M^{\prime} \backslash M} \varphi(x) \delta x \simeq 0$. Similarly we prove that $\sum_{x \in M \backslash M^{\prime}} \varphi(x) \delta x \simeq 0$. Hence $\sum_{x \in M \triangle M^{\prime}} \varphi(x) \delta x \simeq 0$.

(2) Let $Q=[a \ldots b] \backslash(C \bigcup M)$. Then $Q$ is an internal set and $\lambda Q \simeq b-a$. Let $\eta \subseteq Q$ such that $\lambda \eta \simeq 0$. Put $\sigma=\sum_{x \in \eta} \varphi(x) \delta x$. Suppose $\sigma \gtrsim 0$. If there exists a standard $h_{n} \in H$ and an internal interval $J \subset$ hal $\left(\mathrm{h}_{\mathrm{n}}\right)$ such that $\sum_{x \in \eta \cap J} \varphi(x) \delta x \nsucceq 0$, the atomic contribution of $\varphi$ to its discrete integral would be larger than $A$, a contradiction. Hence $\sum_{x \in \eta \cap J} \varphi(x) \delta x \simeq 0$ for every internal interval $J \subset$ hal $\left(\mathrm{h}_{\mathrm{n}}\right)$ such that $h_{n} \in H$ is standard. Applying the Principle of Cauchy we may find for each standard $n \in \mathbb{N}$ an internal interval $K \supset$ hal $\left(\mathrm{h}_{\mathrm{n}}\right)$ such that $\sum_{x \in K \cap \eta} \varphi(x) \delta x \leq \sigma / 2^{n+1}$. By the Extension Principle and the Principle of Cauchy we may find an unlimited integer $\nu$ and an internal sequence $\left(K_{n}\right)_{n \leq \nu}$ such that $K_{n} \supset$ hal $\left(\mathrm{h}_{\mathrm{n}}\right)$ for all standard $n \in \mathbb{N}$ and $\sum_{n \leq \nu} \sum_{x \in K_{n} \cap \eta} \varphi(x) \delta x \leq$ $\sum_{n \leq \nu} \sigma / 2^{n+1} \leq \sigma / 2$. Hence $\sum_{x \in\left(\cup_{n \leq \nu} K_{n}\right) \cap \eta} \varphi(x) \delta x \leq \sigma / 2$, which implies that $\sum_{x \in \cap \eta \backslash\left(\cup_{n \leq \nu} K_{n}\right)} \varphi(x) \delta x \geq \sigma / 2$. Hence the singular contribution to the discrete integral $I$ of $\varphi$ would be larger than $S$, a contradiction. We conclude that $\varphi$ is $S$-integrable on $Q$.

By definition of $R$, one has $\sum_{x \in Q} \varphi(x) \delta x \lesssim R$. Suppose $\sum_{x \in Q} \varphi(x) \delta x \succsim R$. Then there exists some internal set $D \subseteq[a \ldots b]$ such that $\varphi$ is $S$-integrable on $D$ and $\sum_{x \in Q} \varphi(x) \delta x \lesssim \sum_{x \in D} \varphi(x) \delta x$. Because $\lambda Q \simeq b-a$, it holds that $\lambda(D \backslash Q) \simeq 0$. Because $\varphi$ is $S$-integrable on $D \backslash Q$,

$$
\sum_{x \in D} \varphi(x) \delta x=\sum_{x \in D \cap Q} \varphi(x) \delta x+\sum_{x \in D \backslash Q} \varphi(x) \delta x \lesssim \sum_{x \in Q} \varphi(x) \delta x .
$$

So we derived a contradiction. Hence $\sum_{x \in Q} \varphi(x) \delta x \simeq R$.

Assume $Q^{\prime} \subseteq[a \ldots b]$ has the same properties, as prescribed. Since $Q \triangle Q^{\prime}=$ $Q \cap\left(M^{\prime} \cup C^{\prime}\right) \cup Q^{\prime} \cap(M \cup C)$ it is the union of a set of infinitesimal measure within $Q$ and a set of infinitesimal measure within $Q^{\prime}$. By the above $\sum_{x \in Q \triangle Q^{\prime}} \varphi(x) \delta x$ is the sum of two infinitesimals, hence is infinitesimal.

Theorem 5.9 Let $\varphi$ be a function of limited accumulation. Then its discrete integral $I$ may be written in the form $I \simeq A+S+R$, where $A$ is the atomic contribution of $\varphi, S$ is the singular contribution of $\varphi$ and $R$ is the regular contribution of $\varphi$. 
Proof Let $C, M$ and $Q$ be as defined in Theorem 5.7 and 5.8. Because $[a \ldots b]=$ $C \cup M \cup Q$ and $C, M$ and $Q$ are two-by-two disjoint,

$$
I=\sum_{x \in C} \varphi(x) \delta x+\sum_{x \in M} \varphi(x) \delta x+\sum_{x \in Q} \varphi(x) \delta x .
$$

The near-equalities $\sum_{x \in C} \varphi(x) \delta x \simeq A, \sum_{x \in M} \varphi(x) \delta x \simeq S$ and $\sum_{x \in Q} \varphi(x) \delta x \simeq R$ follow also from Theorem 5.7 and 5.8.

\subsection{Decompositions of the domain}

We decompose the interval of definition $[a \ldots b]$ of the function of limited accumulation in a way which corresponds to the decomposition in values of Theorem 5.9. The decomposition of $[a \ldots b]$ will be done in two ways: one into internal sets and one into external sets. As for the first, we adapt the decomposition $[a \ldots b]=C \cup M \cup Q$ of Theorem 5.7 and 5.8, in order to obtain a slightly more natural decomposition.

Theorem 5.10 Let $\varphi$ be a function of limited accumulation. Then $[a \ldots b]=C \cup M \cup Q$, where $C, M$ and $Q$ are internal and two-by-two disjoint, $\lambda C \simeq \lambda M \simeq 0$ and $\varphi$ takes only unlimited values on $C \cup M$, such that $\sum_{x \in C} \varphi(x) \delta x \simeq A, \sum_{x \in M} \varphi(x) \delta x \simeq S$ and $\sum_{x \in Q} \varphi(x) \delta x \simeq R$.

Proof By Theorem 5.9 there exist internal sets $C, M$ and $Q$, which are two-by-two disjoint, satisfy $\lambda C \simeq \lambda M \simeq 0$ and are such that $\sum_{x \in C} \varphi(x) \delta x \simeq A, \sum_{x \in M} \varphi(x) \delta x \simeq S$ and $\sum_{x \in Q} \varphi(x) \delta x \simeq R$. Define for $n \in \mathbb{N}$

$$
M_{n}=\{x \in M \mid \varphi(x) \geq n\} .
$$

Then $\sum_{x \in M_{n}} \varphi(x) \delta x \simeq S$ for all standard $n \in \mathbb{N}$. By Robinson's Lemma there exists unlimited $\nu \in \mathbb{N}$ such that still $\sum_{x \in M_{\nu}} \varphi(x) \delta x \simeq S$. Put $M^{\prime}=M_{\nu}$. Then $\varphi$ takes only unlimited values on $M^{\prime}$ and $\sum_{x \in M^{\prime}} \varphi(x) \delta x \simeq \sum_{x \in M} \varphi(x) \delta x \simeq S$. In the same way we obtain $C^{\prime} \subseteq C$ such that $\varphi$ takes only unlimited values on $C^{\prime}$ and $\sum_{x \in C^{\prime}} \varphi(x) \delta x \simeq \sum_{x \in C} \varphi(x) \delta x \simeq A$. Put $Q^{\prime}=[a \ldots b] \backslash M^{\prime} \cup C^{\prime}$. Then $\sum_{x \in Q^{\prime}} \varphi(x) \delta x \simeq \sum_{x \in Q} \varphi(x) \delta x \simeq R$. By construction $C^{\prime}, M^{\prime}$ and $Q^{\prime}$ are two-by-two disjoint with $\lambda C^{\prime} \simeq \lambda M^{\prime} \simeq 0$.

The internal decomposition of Theorem 5.10 is unique up to sets of infinitesimal measure. The decomposition may be transformed into a "canonical" external decomposition, which is unique. Generically spoken, the contributions to the discrete integral $I$ on these sets cannot be represented by real numbers. However, they may be given in terms of the 
external numbers of Koudjeti and van den Berg [12]. An example of an external number is the external set of all infinitesimals $\oslash$. The external set of external numbers is an ordered structure of particular external intervals of $\mathbb{R}$, with strong algebraic properties. Using the notation of external numbers the following theorem holds.

Theorem 5.11 Let $\varphi$ be a function of limited accumulation. Define

$$
\begin{aligned}
\gamma & =\left\{x \in[a \ldots b] \mid \sum_{y \simeq x} \varphi(y) \delta x>\oslash\right\} \\
\mu & =\left\{x \in[a \ldots b] \mid \sum_{y \simeq x} \varphi(y) \delta x=\oslash, \varphi(x) \simeq+\infty\right\} \\
\theta & =\left\{x \in[a \ldots b] \mid \sum_{y \simeq x} \varphi(y) \delta x=\oslash, \varphi(x) \text { limited. }\right\}
\end{aligned}
$$

Then $\gamma, \mu$ and $\theta$ are two-by-two disjoint and $[a \ldots b]=\gamma \cup \mu \cup \theta$. Moreover there exists an internal set $C \subseteq \gamma$ such that $\sum_{x \in C} \varphi(x) \delta x \simeq A$, an internal set $M \subseteq \mu$ such that $\sum_{x \in M} \varphi(x) \delta x \simeq S$ and for all standard $\epsilon>0$ there exists an internal set $P \subseteq \theta$ such that $\sum_{x \in P} \varphi(x) \delta x \geq R-\epsilon$.

Proof By definition the external sets are two-by-two disjoint and fill up the interval $[a \ldots b]$. The set $C$ of Theorem 5.10 is contained in $\gamma$ and it holds that $\sum_{x \in C} \varphi(x) \delta x \simeq A$. Similarly, the set $M$ of Theorem 5.10 is contained in $\mu$ and it holds that $\sum_{x \in M} \varphi(x) \delta x \simeq$ $S$. For the set $Q=[a \ldots b] \backslash(C \cup M)$ of Theorem 5.10 it holds that $\sum_{x \in Q} \varphi(x) \delta x \simeq R$. We define $\psi=\varphi_{\mid Q}$. For each $n \in \mathbb{N}$ we let $\psi^{(n)}$ be the function $\psi$ truncated at $n$, ie $\psi^{(n)}(x)=\psi(x)$ if $\psi(x) \leq n$ and $\psi^{(n)}(x)=0$ if $\psi(x)>n$, and let $P_{n} \subseteq Q$ be the support of $\psi^{(n)}$. Observe that $\psi$ is regular on $Q$. We claim that $\sum_{x \in Q} \psi^{(\nu)}(x) \delta x \simeq R$ for all $\nu \simeq+\infty$. If not, let $\mu \simeq+\infty$ be such that $\sum_{x \in Q} \psi^{(\mu)}(x) \delta x \gtrsim R$. Then $\sum_{x \in Q}\left(\psi(x)-\psi^{(\mu)}(x)\right) \delta x \geq \mu \lambda\left(Q-P_{\mu}\right) \gtrsim 0$. If $\lambda\left(Q-P_{\mu}\right) \gtrsim 0, \sum_{x \in Q}(\psi(x)-$ $\left.\psi^{(\mu)}(x)\right) \delta x \simeq+\infty$, a contradiction with the fact that $\psi-\psi^{(\mu)} \leq \psi$ is of limited accumulation. If $\lambda\left(Q-P_{\mu}\right) \simeq 0$, the function $\psi-\psi^{(\mu)}$ is singular on $Q-P_{\mu}$, hence also $\psi \geq \psi-\psi^{(\mu)}$, in contradiction with the fact that $\psi$ is assumed to be regular. This proves the claim. Let $\epsilon>0$ be standard. One obtains with the aid of the Principle of Cauchy that $\sum_{x \in Q} \psi^{(n)}(x) \delta x=\sum_{x \in P_{n}} \psi(x) \delta x \geq S-\epsilon$ for some standard $n \in \mathbb{N}$. Noting that $P_{n} \subseteq \theta$, this proves the theorem.

Observe that in general there may not exist an internal set $P \subseteq \theta$ such that $\sum_{x \in P} \varphi(x) \delta x \simeq$ $R$. A counterexample is given by the function $f:] 0 \ldots 1] \rightarrow \mathbb{R}^{+}$defined by $f(x)=1 / \sqrt{x}$. 


\subsection{Decomposition of the function}

We write the function $\varphi$ of limited accumulation itself as the sum of three functions which correspond to the atomic part, the singular part and the regular part of its discrete integral.

Theorem 5.12 Let $\varphi:[a \ldots b] \rightarrow \mathbb{R}^{+}$be a function of limited accumulation. Then there exist functions $\varphi_{A}, \varphi_{S}, \varphi_{R}:[a \ldots b] \rightarrow \mathbb{R}^{+}$with disjoint supports such that $\varphi_{A}$ is atomic, $\varphi_{S}$ is singular, $\varphi_{R}$ is regular and $\varphi=\varphi_{A}+\varphi_{S}+\varphi_{R}$ and its discrete integrals satisfy

$$
\begin{aligned}
& \sum_{x \in[a \ldots b]} \varphi_{A}(x) \delta x \simeq A \\
& \sum_{x \in[a \ldots b]} \varphi_{S}(x) \delta x \simeq S \\
& \sum_{x \in[a \ldots b]} \varphi_{R}(x) \delta x \simeq R .
\end{aligned}
$$

Moreover, let $\varphi_{A}^{\prime}, \varphi_{S}^{\prime}, \varphi_{R}^{\prime}$ be a second decomposition into an atomic function $\varphi_{A}^{\prime}$, a singular function $\varphi_{S}^{\prime}$ and a regular function $\varphi_{R}^{\prime}$ with disjoints supports. Then

$$
\begin{aligned}
\sum_{x \in[a \ldots b]}\left|\varphi_{A}^{\prime}(x)-\varphi_{A}(x)\right| \delta x & \simeq \sum_{x \in[a \ldots b]}\left|\varphi_{S}^{\prime}(x)-\varphi_{S}(x)\right| \delta x \\
& \simeq \sum_{x \in[a \ldots b]}\left|\varphi_{R}^{\prime}(x)-\varphi_{R}(x)\right| \delta x \simeq 0 .
\end{aligned}
$$

Proof Consider the decomposition of $[a \ldots b]$ into internal sets $C, M$ and $Q$ as given by Theorem 5.7 and 5.8. Define $\varphi_{A}=\left.\varphi\right|_{C}, \varphi_{S}=\left.\varphi\right|_{M}$ and $\varphi_{R}=\left.\varphi\right|_{Q}$. Then $\varphi_{A}$ is atomic, $\varphi_{S}$ is singular and $\varphi_{R}$ is regular. Hence $\varphi_{A}, \varphi_{S}, \varphi_{R}$ is the required decomposition. Let $\varphi_{A}^{\prime}, \varphi_{S}^{\prime}, \varphi_{R}^{\prime}$ be a second decomposition satisfying the properties of the theorem. Let $C^{\prime}$ be the support of $\varphi_{A}^{\prime}, M^{\prime}$ be the support of $\varphi_{S}^{\prime}$ and $Q^{\prime}$ be the support of $\varphi_{R}^{\prime}$, supposed disjoint and covering $[a \ldots b]$. Observe that $C^{\prime}$ and $M^{\prime}$ have infinitesimal measure. Clearly $\sum_{x \in C^{\prime}} \varphi_{A}^{\prime}(x) \delta x \lesssim A$ and $\sum_{x \in Q^{\prime}} \varphi_{R}^{\prime}(x) \delta x \lesssim R$.

Suppose firstly that $\sum_{x \in C^{\prime}} \varphi_{A}^{\prime}(x) \delta x \succsim A$. Then there exists a gathering point $h$ and an internal interval $J \subset$ hal $(\mathrm{h}) \cap \mathrm{C}$ such that $\sum_{x \in C^{\prime} \cap J} \varphi_{A}^{\prime}(x) \delta x \gtrsim \alpha_{h}$, while $\sum_{x \in J} \varphi(x) \delta x \simeq \alpha_{h}$. Then $\sum_{x \in J}\left(\varphi_{S}^{\prime}(x)+\varphi_{R}^{\prime}(x)\right) \delta x \gtrsim 0$, meaning that $\varphi_{S}^{\prime}(x)$ or $\varphi_{R}^{\prime}(x)$ cannot be of infinitesimal accumulation, a contradiction. Hence

$$
\sum_{x \in C^{\prime}} \varphi_{A}^{\prime}(x) \delta x \simeq A
$$


observe that if $h$ is a gathering point, we also may conclude that $\sum_{x \in C^{\prime} \cap J} \varphi_{A}^{\prime}(x) \delta x \simeq \alpha_{h}$ for sufficiently large internal intervals $J \subseteq$ hal(h) $\cap \mathrm{C}$. As a consequence of the latter

$$
\sum_{x \in C^{\prime} \cap C} \varphi(x) \delta x \simeq \sum_{h \in H} \alpha_{h}=A .
$$

Secondly,

$$
\sum_{x \in Q^{\prime}} \varphi_{R}^{\prime}(x) \delta x=\sum_{x \in Q^{\prime} \cap C} \varphi_{R}^{\prime}(x) \delta x+\sum_{x \in Q^{\prime} \cap M} \varphi_{R}^{\prime}(x) \delta x+\sum_{x \in Q^{\prime} \cap Q} \varphi_{R}^{\prime}(x) \delta x,
$$

where $\sum_{x \in Q^{\prime} \cap C} \varphi_{R}^{\prime}(x) \delta x \simeq \sum_{x \in Q^{\prime} \cap M} \varphi_{R}^{\prime}(x) \delta x \simeq 0$, being discrete integrals of a regular function over sets with infinitesimal measure. Hence

$$
\sum_{x \in Q^{\prime}} \varphi_{R}^{\prime}(x) \delta x \simeq \sum_{x \in Q^{\prime} \cap Q} \varphi_{R}^{\prime}(x) \delta x \simeq \sum_{x \in Q^{\prime} \cap Q} \varphi_{R}(x) \delta x \lesssim \sum_{x \in Q} \varphi_{R}(x) \delta x .
$$

In the same way we show that $\sum_{x \in Q} \varphi_{R}(x) \delta x \lesssim \sum_{x \in Q^{\prime}} \varphi_{R}^{\prime}(x) \delta x$. Hence

$$
\sum_{x \in Q^{\prime}} \varphi_{R}^{\prime}(x) \delta x \simeq \sum_{x \in Q} \varphi_{R}(x) \delta x \simeq R .
$$

Then it follows from (12), (14) and Theorem 5.9 that $\sum_{x \in M^{\prime}} \varphi_{S}^{\prime}(x) \delta x \simeq S$.

To prove the remaining part of the theorem, we prove first the near-equalities

$$
\sum_{x \in C \triangle C^{\prime}} \varphi_{A}(x) \delta x \simeq \sum_{x \in C \triangle C^{\prime}} \varphi_{A}^{\prime}(x) \delta x \simeq 0
$$

$$
\sum_{x \in M \triangle M^{\prime}} \varphi_{S}(x) \delta x \simeq \sum_{x \in M \triangle M^{\prime}} \varphi_{S}^{\prime}(x) \delta x \simeq 0
$$

$$
\sum_{x \in Q \triangle Q^{\prime}} \varphi_{R}(x) \delta x \simeq \sum_{x \in Q \triangle Q^{\prime}} \varphi_{R}^{\prime}(x) \delta x \simeq 0 .
$$

As for (15), applying (13) we find

$$
\begin{aligned}
\sum_{x \in C \triangle C^{\prime}} \varphi_{A}(x) \delta x & =\sum_{x \in C \backslash C^{\prime}} \varphi_{A}(x) \delta x \\
& =\sum_{x \in C} \varphi_{A}(x) \delta x-\sum_{x \in C \cap C^{\prime}} \varphi_{A}(x) \delta x \\
& \simeq A-\sum_{x \in C \cap C^{\prime}} \varphi(x) \delta x \simeq A-A=0 .
\end{aligned}
$$

Journal of Logic \& Analysis 9:2 (2017) 
In an analogous way we derive that $\sum_{x \in C \triangle C^{\prime}} \varphi_{A}^{\prime}(x) \delta x \simeq 0$. As for (16), we use the fact that $\lambda M \simeq 0$ and $\varphi_{R}^{\prime}$ is regular to obtain

$$
\begin{aligned}
\sum_{x \in M \triangle M^{\prime}} \varphi_{S}(x) \delta x & =\sum_{x \in M \backslash M^{\prime}} \varphi_{S}(x) \delta x \\
& =\sum_{x \in M \backslash M^{\prime}} \varphi_{A}^{\prime}(x) \delta x+\sum_{x \in M \backslash M^{\prime}} \varphi_{R}^{\prime}(x) \delta x \\
& \simeq \sum_{x \in C^{\prime} \backslash(C \cup R)} \varphi_{A}^{\prime}(x) \delta x \\
& \leq \sum_{x \in C^{\prime} \backslash C} \varphi_{A}^{\prime}(x) \delta x \\
& =\sum_{x \in C^{\prime} \Delta C} \varphi_{A}^{\prime}(x) \delta x \simeq 0 .
\end{aligned}
$$

The near-equality $\sum_{x \in M \triangle M^{\prime}} \varphi_{S}^{\prime}(x) \delta x \simeq 0$ is derived analogously. As for (17), we use (15) and (16) to prove that

$$
\begin{aligned}
\sum_{x \in Q \triangle Q^{\prime}} \varphi_{R}(x) \delta x & =\sum_{x \in Q \backslash Q^{\prime}} \varphi_{R}(x) \delta x \\
& =\sum_{x \in Q \cap C^{\prime}} \varphi_{R}(x) \delta x+\sum_{x \in Q \cap M^{\prime}} \varphi_{R}(x) \delta x \\
& =\sum_{x \in Q \cap C^{\prime}} \varphi_{A}^{\prime}(x) \delta x+\sum_{x \in Q \cap M^{\prime}} \varphi_{S}^{\prime}(x) \delta x \\
& =\sum_{x \in C^{\prime} \backslash(M \cup C)} \varphi_{A}^{\prime}(x) \delta x+\sum_{x \in M^{\prime} \backslash(M \cup C)} \varphi_{S}^{\prime}(x) \delta x \\
& \lesssim \sum_{x \in C^{\prime} \backslash C} \varphi_{A}^{\prime}(x) \delta x+\sum_{x \in M^{\prime} \backslash M} \varphi_{S}^{\prime}(x) \delta x \simeq 0,
\end{aligned}
$$

with an analogous derivation of $\sum_{x \in Q \triangle Q^{\prime}} \varphi_{R}^{\prime}(x) \delta x \simeq 0$. 
To finish the proof, using (15)

$$
\begin{aligned}
& \sum_{x \in[a \ldots b]}\left|\varphi_{A}^{\prime}(x)-\varphi_{A}(x)\right| \delta x \\
= & \sum_{x \in C \cap C^{\prime}}\left|\varphi_{A}^{\prime}(x)-\varphi_{A}(x)\right| \delta x+\sum_{x \in C \triangle C^{\prime}}\left|\varphi_{A}^{\prime}(x)-\varphi_{A}(x)\right| \delta x \\
= & \sum_{x \in C \triangle C^{\prime}}\left|\varphi_{A}^{\prime}(x)-\varphi_{A}(x)\right| \delta x \\
\leq & \sum_{x \in C \triangle C^{\prime}}\left|\varphi_{A}^{\prime}(x)\right| \delta x+\sum_{x \in C \triangle C^{\prime}}\left|\varphi_{A}(x)\right| \delta x \\
= & \sum_{x \in C \triangle C^{\prime}} \varphi_{A}^{\prime}(x) \delta x+\sum_{x \in C \triangle C^{\prime}} \varphi_{A}(x) \delta x \\
\simeq & 0 .
\end{aligned}
$$

One shows in analogous way that

$$
\sum_{x \in[a \ldots b]}\left|\varphi_{S}^{\prime}(x)-\varphi_{S}(x)\right| \delta x \simeq 0
$$

and

$$
\sum_{x \in[a \ldots b]}\left|\varphi_{R}^{\prime}(x)-\varphi_{R}(x)\right| \delta x \simeq 0
$$

Example 5.13 Assume $\frac{1}{\sqrt{\delta x}} \in \mathbb{N}$. Consider the function $f: \quad\left[0 \ldots 1\left[\rightarrow \mathbb{R}^{+}\right.\right.$defined by

$$
f(x)=\left\{\begin{array}{cc}
\frac{2 x}{\sqrt{\delta x}} & \frac{x}{\sqrt{\delta x}} \in \mathbb{N} \\
0 & \frac{x}{\sqrt{\delta x}} \notin \mathbb{N} .
\end{array}\right.
$$

Here we may take $C=\emptyset, M=\mathbb{N} \sqrt{\delta x} \cap\left[0 \ldots 1\left[\right.\right.$ and $Q=\left[0 \ldots 1\left[\backslash M\right.\right.$. Then only $f_{S}$ is non-zero. Let us consider $M$ as a near-interval with equally spaced points at distance $\sqrt{\delta x}$ and denote it by $M=[0 . .1[$. Then

$$
S \simeq \sum_{x \in M} f_{S}(x) \delta x=\sum_{x \in M} \frac{2 x}{\sqrt{\delta x}} \delta x=\sum_{x \in[0 . .1[} 2 x \sqrt{\delta x} \simeq \int_{0}^{1} 2 x d x=1,
$$

hence $S=1$, being standard.

Example 5.14 Let $\varphi:[0 \ldots 2] \rightarrow \mathbb{R}$ be defined by

$$
\varphi(x)=\left\{\begin{array}{cc}
\frac{1}{\delta x} & x=1 \\
x & x \neq 1 .
\end{array}\right.
$$


We may take $C=\{1\}, M=\emptyset$ and $R=[0 \ldots 1[\backslash\{1\}$, and we have

$$
A=\varphi_{A}(1) \delta x=\triangle_{1}(1) \delta x=\frac{1}{\delta x} \delta x=1 .
$$

Also

$$
R \simeq \sum_{x \in Q} \varphi_{R}(x) \delta x=\sum_{x \in[0 \ldots 2\lceil\backslash\{1\}} \varphi_{R}(x) \delta x \simeq \sum_{x \in[0 \ldots 2[} x \delta x \simeq \int_{0}^{2} x d x=2,
$$

hence $R=2$, being standard.

Example 5.15 Assume $\frac{1}{\delta x^{1 / 8}} \in \mathbb{N}$. Let $\left.\varphi:\right] 0 \ldots 1\left[\rightarrow \mathbb{R}^{+}\right.$be defined by $\varphi(x)=$ $g(x)+f(x)+w(x)$, with $g$ defined as in (1), $f$ defined as in Example 5.13 and $w$ defined by $w(x)=1 / \sqrt{x}$. We may take $C=] 0 \ldots \delta x^{1 / 8}\left[, M=\mathbb{N} \sqrt{\delta x} \cap\left[\delta x^{1 / 8} \ldots 1[\right.\right.$, and $Q=\left[\delta x^{1 / 8} \ldots 1[\backslash M\right.$. Then it follows from Example 4.10 that

$$
\begin{aligned}
A & \simeq \sum_{10 \ldots \delta x^{1 / 8}[} \varphi_{A}(x) \delta x=\sum_{0<x<\delta x^{1 / 8}} \frac{e^{\frac{-x^{2}}{2 \sqrt{\delta x}}}}{\sqrt{2 \pi \sqrt{\delta x}}} \delta x \\
& \simeq \frac{1}{2} \sum_{-\delta x^{1 / 8}<x<\delta x^{1 / 8}} \frac{e^{\frac{-x^{2}}{2 \sqrt{\delta x}}}}{\sqrt{2 \pi \sqrt{\delta x}}} \delta x \simeq \frac{1}{2} .
\end{aligned}
$$

Let us use the notation $[c . . d]$ for near intervals from limited numbers $c$ to $d$ with steps $\sqrt{\delta x}$, as suggested by Example 5.13. Then

$$
S \simeq \sum_{x \in M} \varphi_{S}(x) \delta x=\sum_{x \in\left[\delta x^{1 / 8} . .1[\right.} 2 x \sqrt{\delta x} \simeq \sum_{x \in] 0 . .1[} 2 x \sqrt{\delta x} \simeq \int_{0}^{1} 2 x d x=1 .
$$

Finally

$$
R \simeq \sum_{x \in Q} \varphi_{R}(x) \delta x \simeq \sum_{x \in\left[\delta x^{1 / 8} \ldots 1[\backslash M\right.} \frac{1}{\sqrt{x}} \delta x \simeq \sum_{x \in] 0 \ldots 1[} \frac{1}{\sqrt{x}} \delta x \simeq \int_{0}^{1} \frac{1}{\sqrt{x}} d x=2 .
$$

We have $A=\frac{1}{2}, S=1$ and $R=2$, because $A, S$ and $R$ are standard. The discrete integral $I$ of $\varphi$ over ]0...1[ amounts to $I \simeq A+S+R=3 \frac{1}{2}$.

The above examples illustrate that a singular or an atomic function may very well be regular after an appropriate rescaling, with discrete integral nearly equal to a Riemann integral. 


\subsection{Decomposition of the discrete primitive}

In this final section we add to the previous decompositions a decomposition of the discrete primitive of a function of limited accumulation. We start by recalling some definitions. As before we suppose that the discrete functions $\varphi$ are non-negative and defined on a near-interval $[a \ldots b]$. For $x \in[a \ldots b$ [ we define the difference function $\delta \varphi$ by $\delta \varphi(x)=\varphi(x+\delta x)-\varphi(x)$.

Definition 5.16 A function $\varphi$ is said to be absolutely $S$-continuous if for all $N \subseteq[a \ldots b[$ with $\lambda N \simeq 0$

$$
\sum_{x \in N} \delta \varphi(x) \simeq 0
$$

The shadow of an absolutely $S$-continuous function with limited values is well-defined and absolutely continuous (Hurd and Loeb [11]).

Definition 5.17 A function $\varphi$ is said to be nearly everywhere constant, if there exists a set $N \subseteq[a \ldots b]$ with $\lambda N \simeq b-a$ such that $\varphi(x)=\varphi(x+\delta x)$ for all $x$ such that $x, x+\delta x \in N$.

A discrete Dirac function is an example of a nearly everywhere constant function.

Definition 5.18 Let $r \in{ }^{\circ}[a, b]$ and $s_{r} \neq 0$ be standard. A function $\varphi$ has a jump in $r$ of width $s_{r}$ if there exist $y, z \in[a \ldots b]$ with $y, z \simeq r$ and $y<x$, such that

$$
\varphi(\zeta)-\varphi(\eta) \simeq s_{r}
$$

for all $\eta, \zeta \in[a \ldots b]$ such that $\eta, \zeta \simeq r, \eta \leq y$ and $\zeta \geq z$.

Definition 5.19 A function $\varphi$ with exactly one jump $s_{r}$ in some point $r \in{ }^{\circ}[a, b]$, of type

$$
\varphi(x)= \begin{cases}0 & a \leq x<\rho \\ \sigma & \rho \leq x \leq b\end{cases}
$$

where $\rho \in[a \ldots b], \rho \simeq r$ and $\sigma \in \mathbb{R}, \sigma \simeq s_{r}$, will be called a discrete function of Heaviside of width $s_{r}$.

Definition 5.20 A limited function $\varphi:[a \ldots b] \rightarrow \mathbb{R}$ such that there exists an internal set $C \subseteq[a \ldots b]$ of infinitesimal measure such that $\delta \Phi(x)=0$ for $x \in[a \ldots b] \backslash C$ and such that it is absolutely $S$-continuous on every internal set $D \subseteq C$ of $[a \ldots b]$ which does not touch any halo of the jumping points, will be called a jump-function. 
Observe that the discrete primitive of a discrete Dirac function is a discrete Heaviside function with width of jump equal to 1 .

Generally spoken a gathering point $h$ of a function of limited accumulation, with accumulation number $\alpha_{h}$, corresponds to a jump of its discrete primitive at $h$ with width $s_{h}=\alpha_{h}$ and vice-versa. So next proposition is a consequence of Theorem 4.11.

Proposition 5.21 Let $\Phi$ be a discrete primitive of a function of limited accumulation. Then the external set of its jumps is at most externally countable.

Proposition 5.22 Let $\Phi$ be the discrete primitive of an atomic function $\varphi$ on $[a \ldots b[$. Then $\Phi$ is a jump-function with $\Phi(b) \simeq A$. Conversely, if $\Phi$ is a jump-function on $[a \ldots b]$, with $\Phi(a)=0$, its discrete derivative $\varphi$ is atomic, with atomic contribution $A \simeq \Phi(b)$.

Proof Let $\Phi$ be the discrete primitive of an atomic function $\varphi$. Let $\Gamma$ be the support of $\varphi$. By Theorem 5.7 there exist $\nu \in \mathbb{N}$ and an internal sequence $\left(J_{n}\right)_{n \leq \nu}$ of disjoint intervals such that, with $J_{n}=\left[j_{n} \ldots k_{n}\right.$ [ and $C=\bigcup_{n \leq \nu} J_{n}$, firstly for all standard $n$ $\in \mathbb{N}$ such $\alpha_{h_{n}} \neq 0$ it holds that $\Phi\left(k_{n}\right)-\Phi\left(j_{n}\right) \simeq \alpha_{h_{n}}$, secondly $\lambda C \simeq 0$, and thirdly $\sum_{x \in C} \varphi(x) \delta x \simeq A$. Notice that also $\varphi_{\mid C}$ is atomic, so

$$
\sum_{x \in \Gamma \backslash C} \varphi(x) \delta x \simeq 0
$$

by Theorem 5.12. Hence $\Phi(b)=\sum_{x \in \Gamma} \varphi(x) \delta x \simeq A$. Moreover, let $D \subseteq \Gamma \backslash$ $\cup_{s t n \in \mathbb{N}}$ hal $\left(\mathrm{h}_{\mathrm{n}}\right)$ be internal. Then (18) and Theorem 5.7 imply that

$$
\sum_{x \in D} \delta \Phi(x)=\sum_{x \in D \backslash C} \varphi(x) \delta x+\sum_{x \in D \cap C} \varphi(x) \delta x \simeq 0 .
$$

We conclude that $\Phi$ is a jump-function.

Conversely, let $\Phi$ be a jump-function, with $\Phi(a)=0$, and $\varphi$ be its discrete derivative. Let $\Gamma$ be the support of $\varphi$. Then $\lambda \Gamma \simeq 0$. Because $\Phi(b)$ is limited, the function $\varphi$ is of limited accumulation. The jumping points, say $h$, with width $s_{h}$ of $\Phi$ correspond to gathering points of $\varphi$ with accumulation number $\alpha_{h}=s_{h}$, and the standardized $H$ of the set of jumping points may be arranged into a sequence, so we may use the notation $\alpha_{h_{n}}$ of Notation 5.1. By Theorem 5.7 we may find $\nu \in \mathbb{N}$ and an internal sequence $\left(J_{n}\right)_{n \leq \nu}$ of disjoint intervals such that, with $J_{n}=\left[j_{n} \ldots k_{n}\right.$ [ and $C=\bigcup_{n \leq \nu} J_{n}$, firstly for all standard $n \in \mathbb{N}$ such $\alpha_{h_{n}} \neq 0$ it holds that $\sum_{x \in\left[j_{n} \ldots k_{n}[\right.} \varphi(x) \delta x=\Phi\left(\overline{k_{n}}\right)-\Phi\left(j_{n}\right) \simeq \alpha_{h_{n}}$, secondly 
$\lambda C \simeq 0$, and thirdly $\sum_{x \in C} \varphi(x) \delta x \simeq A$. Now $\sum_{x \in \Gamma} \varphi(x) \delta x=\Phi(b) \gtrsim \sum_{n \in \mathbb{N}} s_{h_{n}}=$ $\sum_{n \in \mathbb{N}} \alpha_{h_{n}}=A$. Let $D \subseteq \Gamma \backslash \cup_{s t n \in \mathbb{N} h a l}\left(\mathrm{~h}_{\mathrm{n}}\right)$. Then

$$
\sum_{x \in D} \varphi(x) \delta x=\sum_{x \in D} \delta \Phi(x) \simeq 0 .
$$

As a consequence $\varphi$ is atomic. Let $\epsilon>0$ be standard. If $n \in \mathbb{N}$ is standard, because $\sum_{x \in J} \varphi(x) \delta x \simeq \alpha_{h_{n}}$ for every internal interval $J$ with $J_{n} \subseteq J \subseteq$ hal $\left(\mathrm{h}_{\mathrm{n}}\right)$, there exists an internal interval $K \supset$ hal $\left(\mathrm{h}_{\mathrm{n}}\right)$ such that $\sum_{x \in K} \varphi(x) \delta x<\alpha_{h_{n}}+\frac{\epsilon}{2^{n}}$. By the Principle of Extension and the Principle of Cauchy there exists some unlimited $\mu \in \mathbb{N}$ and an internal sequence $\left(K_{n}\right)_{n \leq \mu}$ such that $K_{n} \supset$ hal $\left(\mathrm{h}_{\mathrm{n}}\right)$ for all standard $n$ and $\sum_{x \in K_{n}} \varphi(x) \delta x<\alpha_{h_{n}}+\frac{\epsilon}{2^{n}}$ for all $n \leq \mu$. Applying (19) we find

$$
\begin{aligned}
\sum_{x \in \Gamma} \varphi(x) \delta x & =\sum_{x \in \Gamma \cap \cup_{n \leq \mu} K_{n}} \varphi(x) \delta x+\sum_{x \in \Gamma \backslash \cup_{n \leq \mu} K_{n}} \varphi(x) \delta x \\
& \simeq \sum_{x \in \Gamma \cap \cup_{n \leq \mu} K_{n}} \varphi(x) \delta x \\
& \lesssim A+\epsilon .
\end{aligned}
$$

Because $\epsilon$ is arbitrary, we derive that $\sum_{x \in \Gamma} \varphi(x) \delta x \lesssim A$. Combining, we conclude that $\sum_{x \in \Gamma} \varphi(x) \delta x \simeq A \simeq \Phi(b)$.

Proposition 5.23 Let $\varphi$ be a function of limited accumulation and $\Phi$ be its discrete primitive. Then

(1) $\varphi$ is atomic with atomic contribution $A>0$ if and only if $\Phi$ is a jump-function with $\Phi(a)=0$ and $\Phi(b) \simeq A$.

(2) $\varphi$ is singular with singular contribution $S>0$ if and only if $\Phi$ is $S$-continuous and nearly always constant with $\Phi(a)=0$ and $\Phi(b) \simeq S$.

(3) $\varphi$ is regular with regular contribution $R>0$ if and only if $\Phi$ is absolutely $S$-continuous with $\Phi(a)=0$ and $\Phi(b) \simeq R$.

Conversely, the above equivalences continue to hold if $\Phi:[a \ldots b] \rightarrow \mathbb{R}$ is nondecreasing such that $\Phi(a)=0$ and $\Phi(b)$ is limited and $\varphi:[a \ldots b[\rightarrow \mathbb{R}$ defined by $\varphi=\frac{\delta \Phi}{\delta x}$ is its discrete derivative.

Proof (1) This part follows from Proposition 5.22.

(2) Let $\varphi$ be singular and let $\eta \subseteq\left[a \ldots b\right.$ [ be such that $\lambda \eta \simeq 0, \sum_{x \in \eta} \varphi(x) \delta x \simeq S$ and $\varphi$ is zero on $[a \ldots b] \backslash \eta$. This implies that $\Phi$ is nearly always constant, with 
$\Phi(b)=\Phi(b)-\Phi(a) \simeq S$. Let $y, z \in[a \ldots b]$ with $y<z$ and $y \simeq z$. Because $\varphi$ is of infinitesimal accumulation

$$
\Phi(z)-\Phi(y)=\sum_{y \leq x<z} \varphi(x) \delta x \simeq 0 .
$$

hence $\Phi$ is $S$-continuous.

Conversely, if $\Phi(x)=\Phi(x+\delta x)$ for all $x$ such that $x, x+\delta x \in[a \ldots b] \backslash \eta$, with $\lambda \eta \simeq 0$, its discrete derivative $\varphi$ is nearly always zero. Because $\Phi$ is $S$-continuous, by (20) the function $\varphi$ is of infinitesimal accumulation. The function $\varphi$ is singular, for

$$
\sum_{x \in \eta} \varphi(x) \delta x=\Phi(b)-\Phi(a)=\Phi(b) \simeq S .
$$

(3) Let $\varphi$ be regular and $\eta \subseteq\left[a \ldots b\right.$ [ be such that $\lambda \eta \simeq 0$. Then $\sum_{x \in \eta} \varphi(x) \delta x \simeq 0$. So $\sum_{x \in \eta} \delta \Phi(x) \simeq 0$. Hence $\Phi$ is absolutely $S$-continuous on $[a \ldots b]$. Moreover, $\Phi(a)=0$ and

$$
\Phi(b)=\Phi(b)-\Phi(a)=\sum_{x \in[a \ldots b[} \varphi(x) \delta x \simeq R .
$$

Conversely, if $\Phi$ is absolutely $S$-continuous, one has $\sum_{x \in \eta} \varphi(x) \delta x \simeq \sum_{x \in \eta}$ $\delta \Phi(x) \simeq 0$ for all $\eta \subseteq[a \ldots b$ [ such that $\lambda \eta \simeq 0$. Hence $\varphi$ is regular, with $\sum_{x \in[a \ldots b[} \varphi(x) \delta x=\Phi(b)-\Phi(a)=\Phi(b) \simeq R$.

The second part of the proposition amounts to a reformulation of the first part of the proposition.

We end with a decomposition theorem for non-decreasing functions.

Theorem 5.24 Let $\Phi:[a \ldots b] \rightarrow \mathbb{R}$ be a non-decreasing function such that $\Phi(a)$ and $\Phi(b)$ are limited. Then there exist non-negative non-decreasing functions $\Phi_{A}, \Phi_{S}$ and $\Phi_{R}$ such that $\Phi_{A}$ is a jump-function, $\Phi_{S}$ is an $S$-continuous function which is nearly everywhere constant, and $\Phi_{R}$ is absolutely $S$-continuous, such that

$$
\Phi-\Phi(a)=\Phi_{A}+\Phi_{S}+\Phi_{R} .
$$

Moreover, let $I=\Phi(b)-\Phi(a)$, A be the atomic contribution of $\frac{\delta \Phi}{\delta x}$ to $I, S$ its singular contribution to $I$ and $R$ its regular contribution to $I$. Then $\Phi_{A}(b) \simeq A, \Phi_{S}(b) \simeq S$ and $\Phi_{R}(b) \simeq R$. If $\Phi_{A}^{\prime}, \Phi_{S}^{\prime}, \Phi_{R}^{\prime}$ is a second decomposition of $\Phi-\Phi(a)$ into a jump-function $\Phi_{A}^{\prime}$, an $S$-continuous function which is nearly everywhere constant $\Phi_{S}^{\prime}$, and an absolutely $S$-continuous function $\Phi_{R}^{\prime}$, all non-negative and non-decreasing, then $\Phi_{A}^{\prime}(x) \simeq \Phi_{A}(x), \Phi_{S}^{\prime}(x) \simeq \Phi_{S}(x)$ and $\Phi_{R}^{\prime}(x) \simeq \Phi_{R}(x)$ for all $x \in[a \ldots b]$. 
Proof Let $\varphi_{A}, \varphi_{S}, \varphi_{R}$ be the decomposition of $\varphi=\frac{\delta \Phi}{\delta x}$ given by Theorem 5.12. Let $\Phi_{A}, \Phi_{S}$, respectively $\Phi_{R}$ be the discrete primitive of $\varphi_{A}, \varphi_{S}$, respectively $\varphi_{R}$. By Proposition 5.23 the function $\Phi_{A}$ is a jump-function, the function $\Phi_{R}$ is $S$-continuous and nearly everywhere constant and the function $\Phi_{R}$ is absolutely $S$-continuous. Because the supports of $\varphi_{A}, \varphi_{S}$ and $\varphi_{R}$ are disjoint, one has $\Phi-\Phi(a)=\Phi_{A}+\Phi_{S}+\Phi_{R}$. Again by Proposition 5.23 we have $\Phi_{A}(b) \simeq A, \Phi_{S}(b) \simeq S$ and $\Phi_{R}(b) \simeq R$. Let $\Phi_{A}^{\prime}, \Phi_{S}^{\prime}, \Phi_{R}^{\prime}$ be a second decomposition of $\Phi$ into a jump-function $\Phi_{A}^{\prime}$, an $S$-continuous function which is nearly everywhere constant $\Phi_{S}^{\prime}$, and an absolutely $S$-continuous function $\Phi_{R}^{\prime}$, all non-negative and non-decreasing. Observe that $\Phi_{A}(a)=\Phi_{A}^{\prime}(a)=\Phi_{S}(a)=\Phi_{A}^{\prime}(a)=$ $\Phi_{R}(a)=\Phi_{R}^{\prime}(a)=0$. Let $x \in\left[a \ldots b\left[\right.\right.$. Put $\varphi_{A}^{\prime}=\frac{\delta \Phi_{A}^{\prime}}{\delta x}, \varphi_{S}^{\prime}=\frac{\delta \Phi_{S}^{\prime}}{\delta x}$ and $\varphi_{R}^{\prime}=\frac{\delta \Phi_{R}^{\prime}}{\delta x}$. Then by Theorem 5.12

$$
\left|\Phi_{A}^{\prime}(x)-\Phi_{A}(x)\right|=\left|\sum_{a \leq y<x}\left(\varphi_{A}^{\prime}(y)-\varphi_{A}(y)\right) \delta x\right| \simeq 0 .
$$

Hence $\Phi_{A}^{\prime}(x) \simeq \Phi_{A}(x)$. One shows in the same way that $\Phi_{S}^{\prime}(x) \simeq \Phi_{S}(x)$ and $\Phi_{R}^{\prime}(x) \simeq$ $\Phi_{R}(x)$.

\section{References}

[1] R.G. Bartle, A Modern Theory of Integration, Graduate Studies in Mathematics 32, American Mathematical Society (2001).

[2] I.P. van den Berg, Nonstandard Asymptotic Analysis, Springer Lecture Notes in Mathematics 1249 (1987).

[3] I.P. van den Berg, Equations paraboliques et intégrales de chemins finies avec applications financières, Publication pédagogique 32, Université de Nice Sophia-Antipolis (1998).

[4] I.P. van den Berg, Discretizations of higher order and the theorems of Faà di Bruno and DeMoivre-Laplace, Journal of Logic \& Analysis 5:6, p. 1-35 (2013). doi: 10.4115/jla.2013.5.6

[5] J.-L. Callot, Analyse grossière et analyse infinitésimale, in: Actes du Colloque Trajectorien, Obernai, publ. IRMA Strasbourg (1995) 229-237.

[6] C. Canelas, Funções de acumulação limitada, Masters Thesis, University of Évora (2010).

[7] P. Cartier and Y. Perrin, Integration over finite sets, in: F. and M. Diener eds., Nonstandard Analysis in Practice, Springer Universitext (1995) 185-204.

[8] F. Diener and M. Diener, eds., Nonstandard Analysis in Practice, Springer Universitext (1995). 
[9] F. Diener and G. Reeb, Analyse Non Standard, Hermann, Paris (1989).

[10] A.J. Franco de Oliveira and I.P. van den Berg, Matemática Não Standard, Uma introdução com aplicações, Edição Gulbenkian, Lisbon (2007).

[11] A. Hurd and P. Loeb, An introduction to nonstandard real analysis, Pure and Applied Mathematics 118, Academic Press (1985).

[12] F. Koudjeti and I.P. van den Berg, Neutrices, external numbers and external calculus, in: F. and M. Diener eds., Nonstandard Analysis in Practice, Springer Universitext (1995 145-170). doi: 10.1007/978-3-642-57758-1_7

[13] A. Robert, Nonstandard Analysis, John Wiley \& Sons (1988).

[14] A. Robinson, Nonstandard Analysis, $3^{\text {rd }}$ edition, Princeton University Press (1996).

[15] E. Nelson, Internal Set Theory, Bull. Amer. Math. Soc. 83, no. 6 (1977) doi: 10.1090/S0002-9904-1977-14398-X.

[16] E. Nelson, Radically Elementary Probability Theory, Princ. Univ. Press (1987).

[17] J. Sousa Pinto, Métodos Infinitesimais de Análise Matemática, Gulbenkian, Lisbon (2000). English translation by R.F. Hoskins: Infinitesimal methods for Mathematical Analysis, Horwood, Chichester (2004). doi: 10.1533/9780857099501

[18] K.D. Stroyan and J.M. Bayod, Foundations of infinitesimal stochastic analysis. Studies in Logic and the Foundations of Mathematics, 119, North-Holland Publishing Co., Amsterdam (1986).

Department of Mathematics, University of Évora, Colégio Luís Verney, Rua Romão Ramalho 59, 7000-671 Évora, Portugal

ivdb@uevora.pt

Received: 12 February $2016 \quad$ Revised: 24 March 2017 